\title{
An Attachable ECG Sensor Bandage with Planar-Fashionable Circuit Board
}

\author{
Jerald Yoo, Long Yan, Seulki Lee, Hyejung Kim, Binhee Kim, and Hoi-Jun Yoo \\ Dept. of EECS, Korea Advanced Institute of Science and Technology (KAIST) \\ jerald@eeinfo.kaist.ac.kr
}

\begin{abstract}
An attachable ECG sensor adhesive bandage is implemented for continuous ECG monitoring system by using Planar-Fashionable Circuit Board (P-FCB) technology. The sensor patch improves convenience at low cost: it is composed of dry electrodes and an inductor directly screen printed on fabric, and the sensor chip is also directly wire bonded on fabric. The sensor patch is wirelessly powered to remove battery for safety. Dry electrodes minimize skin irritation to enable long term monitoring. The implemented sensor patch successfully demonstrates capturing of ECG signal while dissipating only $12 \mu \mathrm{W}$ power.
\end{abstract}

\section{Introduction}

Nowadays, heart disease is one of the major causes of death around the world. Many heart diseases are chronic, and they require continuous ECG monitoring to prevent more serious stroke or heart attack. Currently, Holter monitor system is widely used for 24-hour heart monitoring and capturing of signals. Although Holter monitor is accurate and powerful way of detecting irregular activities of heart such as arrhythmia, its cumbersome wires (up to 10) make it uncomfortable to carry all day. Moreover, conventional $\mathrm{Ag} / \mathrm{AgCl}$ electrodes may result in skin dermatitis and they suffer from signal degradation due to dehydration [1], so it is unsuitable for long term monitoring.

In this paper, we propose an adhesive ECG sensor bandage to overcome the above problems in long term ECG monitoring. The requirements of the sensor patch for continuous monitoring are: 1) convenience, 2) safety and 3) low cost. For convenience, the sensor is implemented as an adhesive bandage-like patch using Planar-Fashionable Circuit Board (P-FCB) [2-3], so it is attachable and disposable after usage. By P-FCB, silver paste is screen printed on a fabric, and it enables convenient implementation of fabric circuit board with the chip directly integrated onto a cloth. To enhance safety, the sensor adopts dry fabric electrodes instead of conventional $\mathrm{Ag} / \mathrm{AgCl}$ wet electrodes. Also, since

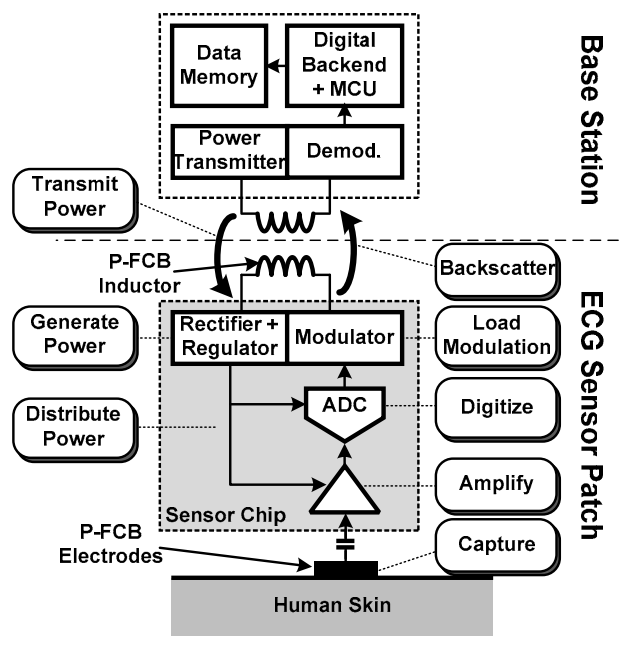

Figure 1. Design architecture of the adhesive bandage ECG sensor patch

the sensor does not have battery within it, safety concern, due to attaching sensor on skin over a long time, is reduced. Finally, for low cost, components such as inductor, electrode, and chip integration are all made by simple and inexpensive P-FCB [2-3].

\section{System Architecture}

Figure 1 illustrates the design architecture of the ECG monitoring system using the proposed adhesive bandage ECG sensor patch. The system is largely composed of two parts: an adhesive bandage ECG sensor patch and a base station.

The proposed ECG sensor patch is powered by an internal rectifier that generates power from incoming HF $(13.56 \mathrm{MHz})$ band carrier, combined with a P-FCB inductor. ECG signal is captured by P-FCB electrodes, amplified, digitized, and fed into an Amplitude-Shift Keying (ASK) modulator that will backscatter the data to the base station. The whole sensor IC is implemented as a single chip. Since the power budget is extremely low, overall power consumption of the sensor chip is suppressed down to $12 \mu \mathrm{W}$ while in operation [4]. The base station could be placed within an underwear or small vest, and it locates and provides 


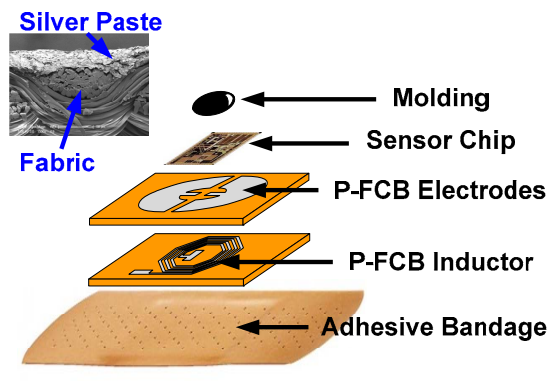

Figure 2. Layered structure of the adhesive bandage ECG sensor by P-FCB

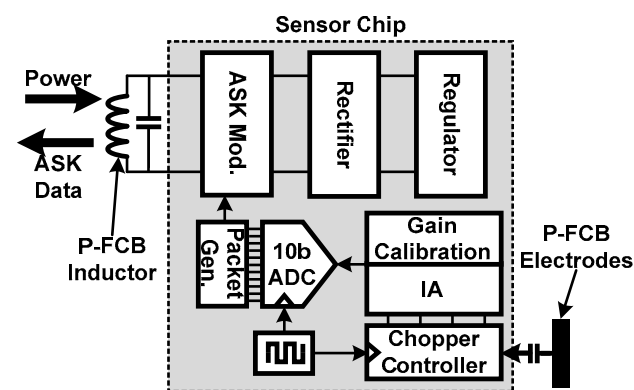

Figure 3. Proposed adhesive bandage ECG sensor architecture

power only to the sensor detected underneath [4]. ECG data backscattered to the carrier will be demodulated and processed.

\section{Realization}

Figure 2 shows the layered structure of the adhesive bandage ECG sensor. A SEM photo of the cross section of the P-FCB is shown on the upper left (1000x magnification). On top of adhesive bandage, an inductor and a pair of electrodes are stacked; a sensor chip is then wire bonded and protected by molding. With the dry electrode, skin irritation and the dehydration effect in wet electrode is minimized. The screen printed process of P-FCB lowers the production cost of the sensor. The sensor can be attached on and removed from skin with ease like a common daily Band-Aid ${ }^{\circledR}$.

Figure 3 is the proposed adhesive bandage ECG sensor architecture. The $2.4 \mathrm{~mm} \times 2.0 \mathrm{~mm}$ sensor chip is composed of a power supply block (rectifier, regulator), an analog front-end (an instrumentation amplifier and an ADC), and subsidiary blocks. The overall power consumption of the sensor chip is $12 \mu \mathrm{W}$ at nominal regulated supply voltage of $1.8 \mathrm{~V}$. Dry electrodes result in more induced noise and more loading effect due to high electrode-skin impedance, and to alleviate the problems, the instrumentation amplifier adopts nested chopper scheme [4].

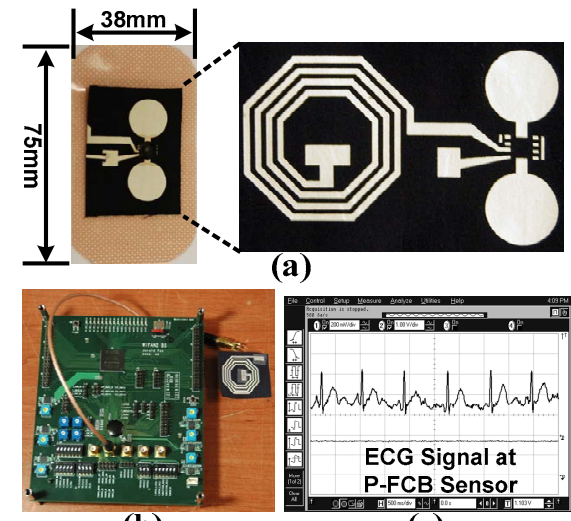

(b)

(c)

Figure 4. (a) Implemented ECG sensor, (b) base station board and (c) the measured ECG signal at the sensor

Figure 4 (a) shows the implemented sensor. Its size is $38 \mathrm{~mm} \times 75 \mathrm{~mm}$ with an octagonal inductor having $0.98 \mu \mathrm{H}$ and $\mathrm{Q}=10.8$ (shown on right with a pair of electrodes). In this work, the base station is implemented as a separate board (Figure 4 (b)) for the detail characterization of the system. Figure 4 (c) is the measured ECG waveform at the sensor amplifier output, while power is provided wirelessly from the base station.

\section{Conclusion}

An adhesive bandage ECG sensor patch is implemented using P-FCB for continuous ECG monitoring system. The sensor is wirelessly powered up, and dry electrodes are adopted for long term monitoring with minimum skin irritation. It achieves low production cost and low power dissipation along with convenience. The entire system is implemented and ECG signal recording is successfully demonstrated.

\section{References}

[1] A. Searle, et al., "A direct comparison of wet, dry and insulating bioelectric recording electrodes," Physiological Measurement, pp. 271-283, vol. 21, 2000.

[2] H. Kim, et al., "A $1.12 \mathrm{~mW}$ Continuous Healthcare Monitor Chip Integrated on A Planar-Fashionable Circuit Board," in IEEE ISSCC Dig. Tech. Papers, pp. 150-151, Feb. 2008.

[3] H. Kim, et al., "A Wearable Fabric Computer by PlanarFashionable Circuit Board Technique," in Proc. $6^{\text {th }}$ Int. Workshop on Body Sensor Network, 2009 (To be published). [4] J. Yoo, et al., "A 5.2mW Self-Configured Wearable Body Sensor Network Controller and a $12 \mu \mathrm{W} 54.9 \%$ Efficiency Wirelessly Powered Sensor for Continuous Health Monitoring System," in IEEE ISSCC Dig. Tech. Papers, pp. 290-291, Feb. 2009. 